\title{
Elevated $\mathrm{HbA} 1 \mathrm{c}$ remains a predominant finding in severe COVID-19 and may be associated with increased mortality in patients requiring mechanical ventilation
}

\author{
Sebastian J. Klein ${ }^{1}$, Timo Mayerhöfer ${ }^{1}$, Dietmar Fries ${ }^{2}$, Christian Preuß Hernández ${ }^{3}$ and Michael Joannidis ${ }^{1^{*}}$ (i) \\ Collaborators
}

\section{Dear Editor,}

We have previously reported unusually high rates of elevated glycated hemoglobin (HbA1c) levels in patients with COVID-19 admitted to an ICU between March 11 and April 29, 2020 [1]. Since then, our Tyrolean multicenter COVID-19 Intensive Care Unit Registry (TyrolCoV-ICU-Reg) [2] has considerably increased, surpassing 500 patients. To re-evaluate our previously reported findings, we included 306 additional patients in this analysis, who were admitted between April 30, 2020 and May 31, 2021, for whom an admission HbA1c was available. Details of our registry have been reported before [2].

Admission HbA1c was now available in 350 patients. We were able to confirm our finding that $\mathrm{HbA1c}$ was elevated (i.e., $\mathrm{HbA1c} \geq 5.7 \%$ ) in most patients $(85.1 \%)$. However, only $31.7 \%$ had a history of diabetes mellitus (DM) or prediabetes (Table 1). Median HbA1c at admission was significantly higher in patients with $\mathrm{HbA1c} \geq 6.5 \%$ and a history of DM than without history of DM (MannWhitney $U \mathrm{p}<0.001)$. Furthermore, a weak correlation for HbA1c and BMI could be established (Pearson $\mathrm{R}=0.27, \mathrm{p}<0.001$ ).
There was a trend toward longer duration of invasive mechanical ventilation (IMV) in patients with higher HbA1c, albeit non-significant. In patients with history of DM, a tendency toward increased mortality associated with elevated HbA1c was observed, but the groups differed considerably in size (Table 1 ). When comparing patients with an $\mathrm{HbA} 1 \mathrm{c} \geq 6.5 \%$ requiring IMV to the rest of our cohort, ICU $\left(40.8 \%\right.$ vs. $\left.21.5 \% ; \mathrm{X}^{2} \mathrm{p}=0.001\right)$ and hospital mortality $\left(42.1 \%\right.$ vs. $\left.24.8 \% ; \mathrm{X}^{2} \mathrm{p}=0.005\right)$ were significantly increased.

In a multivariate logistic regression model including HbA1c, history of DM and IMV, odds ratios (ORs) for hospital death were higher for patients with elevated HbA1c $\geq 6.5 \%$. However, this effect seems to be mainly driven by IMV. A significant association with hospital mortality was shown for treatment with IMV, age and SAPS III score (Table 2).

In this follow-up analysis, including 306 additional cases, we were able to confirm our previous findings [1] of an extremely high incidence of elevated $\mathrm{HbA} 1 \mathrm{c}$ in critically ill COVID-19 patients. While other pre-admission comorbidities (e.g., arterial hypertension) were more

\footnotetext{
*Correspondence: michael.joannidis@i-med.ac.at

${ }^{1}$ Division of Intensive Care and Emergency Medicine, Department

of Internal Medicine, Medical University Innsbruck, Anichstrasse 35, 6020 Innsbruck, Austria

Full list of author information is available at the end of the article
}

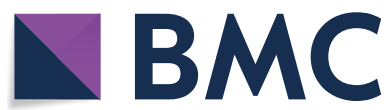

(c) The Author(s) 2021. Open Access This article is licensed under a Creative Commons Attribution 4.0 International License, which permits use, sharing, adaptation, distribution and reproduction in any medium or format, as long as you give appropriate credit to the original author(s) and the source, provide a link to the Creative Commons licence, and indicate if changes were made. The images or other third party material in this article are included in the article's Creative Commons licence, unless indicated otherwise in a credit line to the material. If material is not included in the article's Creative Commons licence and your intended use is not permitted by statutory regulation or exceeds the permitted use, you will need to obtain permission directly from the copyright holder. To view a copy of this licence, visit http://creativecommons.org/licenses/by/4.0/. The Creative Commons Public Domain Dedication waiver (http://creativeco mmons.org/publicdomain/zero/1.0/) applies to the data made available in this article, unless otherwise stated in a credit line to the data. 
Table 1 General characteristics of included patients stratified by history of diabetes mellitus/prediabetes and HbA1C

\begin{tabular}{|c|c|c|c|c|c|c|c|}
\hline & \multirow[t]{2}{*}{ Overall } & \multicolumn{3}{|c|}{ No history of diabetes mellitus/prediabetes } & \multicolumn{3}{|c|}{ History of diabetes mellitus/prediabetes } \\
\hline & & $\mathrm{HbA} 1 \mathrm{c}<5.7 \%$ & $\begin{array}{l}\text { HbA1c } \\
5.7<6.5 \%\end{array}$ & $\mathrm{HbA} 1 \mathrm{c} \geq 6.5 \%$ & $\mathrm{HbA} 1 \mathrm{c}<5.7 \%$ & $\begin{array}{l}\text { HbA1c } \\
5.7<6.5 \%\end{array}$ & $\mathrm{HbA} 1 \mathrm{c} \geq 6.5 \%$ \\
\hline$n$ & 350 & 49 & 138 & 52 & 3 & 18 & 90 \\
\hline $\begin{array}{l}\text { Age [years] } \\
\text { (median [IQR]) }\end{array}$ & $\begin{array}{l}68.00[58.00 \\
76.00]\end{array}$ & $\begin{array}{l}67.00[54.00 \\
77.00]\end{array}$ & $\begin{array}{l}70.00[61.00 \\
77.00]\end{array}$ & $\begin{array}{l}65.00[55.50 \\
75.25]\end{array}$ & $\begin{array}{l}63.00[56.50 \\
68.00]\end{array}$ & $\begin{array}{l}72.00[62.50 \\
74.75]\end{array}$ & $\begin{array}{l}68.00[58.25 \\
76.75]\end{array}$ \\
\hline Male-no. (\%) & $250(71.4)$ & $32(65.3)$ & $102(73.9)$ & $40(76.9)$ & $3(100.0)$ & $11(61.1)$ & $62(68.9)$ \\
\hline $\begin{array}{l}\text { BMI [kg/m2] } \\
\text { (median [IQR]) }\end{array}$ & $\begin{array}{l}27.76[25.14 \\
31.22]\end{array}$ & $\begin{array}{l}25.12[22.10 \\
27.53]\end{array}$ & $\begin{array}{l}27.47[24.97 \\
30.66]\end{array}$ & $\begin{array}{l}29.45[26.41 \\
31.51]\end{array}$ & $\begin{array}{l}25.13[24.18 \\
29.52]\end{array}$ & $\begin{array}{l}28.73[26.93 \\
31.71]\end{array}$ & $\begin{array}{l}29.39[26.51 \\
32.92]\end{array}$ \\
\hline $\begin{array}{l}\text { HbA1c [\%] } \\
\text { (median [IQR]) }\end{array}$ & $6.30[5.90,6.80]$ & $5.40[5.10,5.50]$ & $6.10[5.90,6.20]$ & $6.70[6.60,7.12]$ & $5.50[5.50,5.55]$ & $6.10[5.93,6.30]$ & $7.60[6.80,8.88]$ \\
\hline IMV—no. (\%) & $194(55.4)$ & $28(57.1)$ & $80(58.0)$ & $31(59.6)$ & $1(33.3)$ & $9(50.0)$ & $45(50.0)$ \\
\hline \multicolumn{8}{|l|}{ AKI—no. (\%) } \\
\hline no AKI & $233(67.0)$ & $33(68.8)$ & $94(68.6)$ & $31(59.6)$ & $2(66.7)$ & $12(66.7)$ & $61(67.8)$ \\
\hline KDIGOI & $38(10.9)$ & $4(8.3)$ & $16(11.7)$ & $7(13.5)$ & $0(0.0)$ & $2(11.1)$ & $9(10.0)$ \\
\hline KDIGO II & $18(5.2)$ & $2(4.2)$ & $7(5.1)$ & $2(3.8)$ & $0(0.0)$ & $1(5.6)$ & $6(6.7)$ \\
\hline KDIGO III & $59(17.0)$ & $9(18.8)$ & $20(14.6)$ & $12(23.1)$ & $1(33.3)$ & $3(16.7)$ & $14(15.6)$ \\
\hline RRT—no. (\%) & $55(15.7)$ & $9(18.4)$ & $18(13.0)$ & $10(19.2)$ & $1(33.3)$ & $5(27.8)$ & $12(13.3)$ \\
\hline vv-ECMO & $18(5.1)$ & $4(8.2)$ & $5(3.6)$ & $6(11.5)$ & $0(0.0)$ & $0(0.0)$ & $3(3.3)$ \\
\hline $\begin{array}{l}\text { IMV [days] } \\
\quad \text { (median [IQR]) }\end{array}$ & $14.0[8.0,24.3]$ & $10.5[5.5,20.8]$ & $13.0[8.0,25.3]$ & $\begin{array}{l}15.00[12.5 \\
26.50]\end{array}$ & $7.0[7.0,7.0]$ & $16.0[5.0,29.0]$ & $13.0[8.0,24.0]$ \\
\hline $\begin{array}{l}\text { RRT [days] } \\
\quad \text { (median [IQR]) }\end{array}$ & $11.0[3.0,25.5]$ & $12.0[6.0,13.0]$ & $16.5[3.5,27.5]$ & $13.50[9.5,25.8]$ & $7.0[7.0,7.0]$ & $1.0[1.0,4.0]$ & $4.0[1.8,14.5]$ \\
\hline $\begin{array}{l}\text { ECMO [days] } \\
\quad \text { (median [IQR]) }\end{array}$ & $23.5[12.5,28.8]$ & $15.0[14.0,16.5]$ & $26.0[14.0,26.0]$ & $28.50[16.0,38.0]$ & $N A[N A, N A]$ & $N A[N A, N A]$ & $27.0[19.5,28.5]$ \\
\hline $\begin{array}{l}\text { SAPS III score } \\
\text { (median [IQR]) }\end{array}$ & $55.0[48.0,63.0]$ & $59.0[49.5,69.8]$ & $54.5[49.0,63.3]$ & $52.50[47.0,62.5]$ & $53.0[49.5,61.5]$ & $55.5[50.8,59.3]$ & $55.0[48.0,62.0]$ \\
\hline $\begin{array}{l}\text { Hospital LOS } \\
\text { (median [IQR]) }\end{array}$ & $23.0[14.0,39.5]$ & $26.5[14.0,40.0]$ & $22.0[15.0,35.0]$ & $24.00[12.0,45.5]$ & $44.0[33.5,61.5]$ & $30.5[14.8,45.5]$ & $21.0[13.0,36.5]$ \\
\hline $\begin{array}{l}\text { ICU LOS (median } \\
\text { [IQR]) }\end{array}$ & $11.0[5.0,23.0]$ & $10.0[4.0,23.0]$ & $13.0[7.0,23.0]$ & $16.50[5.0,29.0]$ & $5.0[3.5,10.5]$ & $9.5[6.0,30.5]$ & $10.0[5.0,21.0]$ \\
\hline \multicolumn{8}{|l|}{$\begin{array}{l}\text { Known comor- } \\
\text { bidity*_no. } \\
(\%)\end{array}$} \\
\hline Cardiovascular & $142(40.6)$ & $21(42.9)$ & $56(40.6)$ & $18(34.6)$ & $2(66.7)$ & $12(66.7)$ & $33(36.7)$ \\
\hline $\begin{array}{l}\text { Arterial hyperten- } \\
\text { sion }\end{array}$ & $221(63.1)$ & $26(53.1)$ & $82(59.4)$ & $25(48.1)$ & $3(100.0)$ & $15(83.3)$ & $70(77.8)$ \\
\hline Renal & $78(22.3)$ & $12(24.5)$ & $24(17.4)$ & $10(19.2)$ & $2(66.7)$ & $8(44.4)$ & $22(24.4)$ \\
\hline Liver & $28(8.0)$ & $3(6.1)$ & $12(8.7)$ & $6(11.5)$ & $0(0.0)$ & $1(5.6)$ & $6(6.7)$ \\
\hline $\begin{array}{l}\text { Metastatic } \\
\text { disease }\end{array}$ & $2(0.6)$ & $0(0.0)$ & $0(0.0)$ & $1(1.9)$ & $0(0.0)$ & $0(0.0)$ & $1(1.1)$ \\
\hline $\begin{array}{l}\text { Hematological } \\
\text { malignancy }\end{array}$ & $18(5.1)$ & $3(6.1)$ & $7(5.1)$ & $2(3.8)$ & $0(0.0)$ & $0(0.0)$ & $6(6.7)$ \\
\hline $\begin{array}{l}\text { Non-hematologi- } \\
\text { cal malignancy }\end{array}$ & $28(8.0)$ & $3(6.1)$ & $15(10.9)$ & $3(5.8)$ & $0(0.0)$ & $0(0.0)$ & $7(7.9)$ \\
\hline COPD & $17(4.9)$ & $4(8.2)$ & $8(5.8)$ & $0(0.0)$ & $0(0.0)$ & $1(5.6)$ & $4(4.4)$ \\
\hline Asthma & $50(14.3)$ & $4(8.2)$ & $18(13.0)$ & $8(15.4)$ & $1(33.3)$ & $2(11.1)$ & $17(18.9)$ \\
\hline $\begin{array}{l}\text { Respiratory dis- } \\
\text { ease-others }\end{array}$ & $27(7.7)$ & $2(4.1)$ & $12(8.7)$ & $3(5.8)$ & $0(0.0)$ & $2(11.1)$ & $8(8.9)$ \\
\hline \multicolumn{8}{|l|}{$\begin{array}{l}\text { Diabetes mel- } \\
\text { litus—no. (\%) }\end{array}$} \\
\hline $\begin{array}{l}\text { No pre-known } \\
\text { dysglycemia }\end{array}$ & $239(68.3)$ & $49(100.0)$ & $138(100.0)$ & $52(100.0)$ & $0(0.0)$ & $0(0.0)$ & $0(0.0)$ \\
\hline Prediabetes & $12(3.4)$ & $0(0.0)$ & $0(0.0)$ & $0(0.0)$ & $0(0.0)$ & $4(22.2)$ & $8(8.9)$ \\
\hline DM Type I & $4(1.1)$ & $0(0.0)$ & $0(0.0)$ & $0(0.0)$ & $0(0.0)$ & $2(11.1)$ & $2(2.2)$ \\
\hline
\end{tabular}


Table 1 (continued)

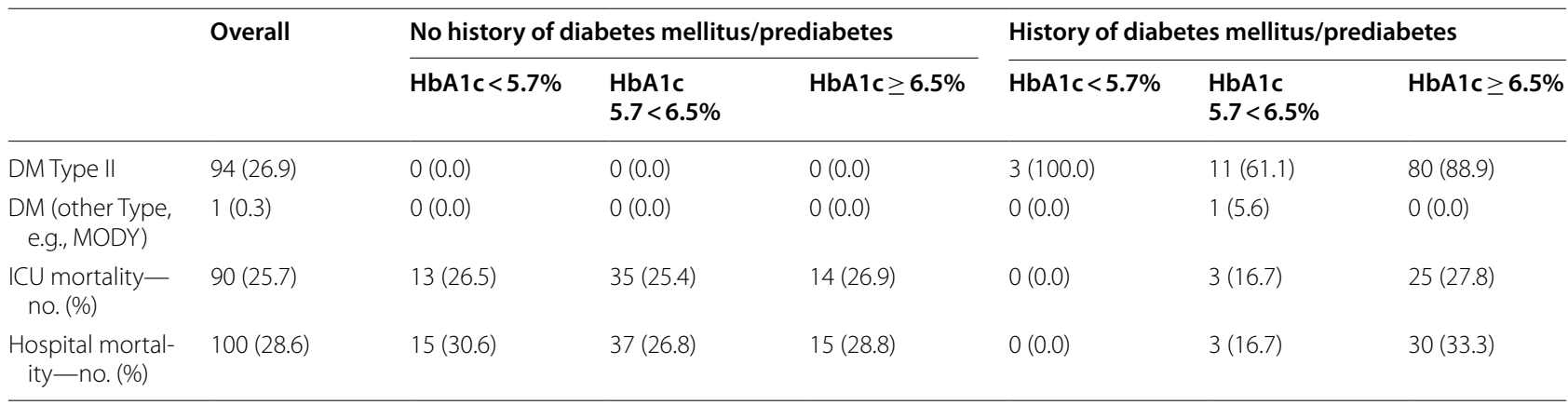

Of 350 included patients, 44 have been previously reported [1]

$I Q R$ interquartile range, $B M I$ body mass index, $H b A 1 c$ glycated hemoglobin, IMV invasive mechanical ventilation, $A K I$ acute kidney injury, $K D I G O$ kidney disease: improving global outcomes, RRT renal replacement therapy, $v v$-ECMO veno-venous extracorporeal membrane oxygenation, SAPS simplified acute physiology score, LOS length of stay, ICU intensive care unit, COPD chronic obstructive pulmonary disease, DM Diabetes mellitus

${ }^{*}$ As mentioned in previous medical documents

Table 2 Logistic regression analysis for prediction of hospital mortality

\begin{tabular}{|c|c|c|c|c|}
\hline & \multicolumn{2}{|c|}{ Univariate analysis } & \multicolumn{2}{|c|}{ Multivariate analysis } \\
\hline & OR $(95 \% \mathrm{Cl})$ & $\mathbf{p}$ & OR $(95 \% \mathrm{Cl})$ & $\mathrm{p}$ \\
\hline Age & $1.08(1.05-1.11)$ & $<0.001$ & $1.07(1.04-1.11)$ & $<0.001$ \\
\hline Sex (male) & $1.29(0.77-2.21)$ & 0.350 & & \\
\hline $\mathrm{BMl}$ & $1.00(0.97-1.05)$ & 0.737 & & \\
\hline Number of comorbidities & $1.29(1.13-1.49)$ & $<0.001$ & $1.24(1.04-1.48)$ & 0.018 \\
\hline SAPS III score & $1.08(1.05-1.11)$ & $<0.001$ & $1.05(1.02-1.08)$ & 0.001 \\
\hline IMV & $3.08(1.87-5.21)$ & $<0.001$ & & \\
\hline \multicolumn{5}{|l|}{ Ref: HbAlc $<6.5$ and no history of DM } \\
\hline $\mathrm{HbA} 1 \mathrm{c}<6.5$ and history of DM & $0.43(0.20-1.35)$ & 0.194 & & \\
\hline $\mathrm{HbA} 1 \mathrm{c} \geq 6.5$ and no history of DM & $1.05(0.52-2.05)$ & 0.883 & & \\
\hline $\mathrm{HbA1c} \geq 6.5$ and history of $\mathrm{DM}$ & $1.30(0.75-2.23)$ & 0.346 & & \\
\hline \multicolumn{5}{|l|}{ Ref: HbAlc $<6.5$ and no history of DM and no IMV } \\
\hline $\mathrm{HbA} 1 \mathrm{c}<6.5$ and history of DM, no IMV & & & $0.30(0.01-2.16)$ & 0.304 \\
\hline $\mathrm{HbA1c} \geq 6.5$ and no history of DM, no IMV & & & $0.31(0.02-1.99)$ & 0.298 \\
\hline $\mathrm{HbA} 1 \mathrm{c} \geq 6.5$ and history of $\mathrm{DM}$, no IMV & & & $2.18(0.71-6.71)$ & 0.168 \\
\hline $\mathrm{HbA} 1 \mathrm{c}<6.5$ and no history of DM, IMV & & & $3.94(1.72-9.66)$ & 0.002 \\
\hline $\mathrm{HbA} 1 \mathrm{c}<6.5$ and history of $\mathrm{DM}$, IMV & & & $0.78(0.04-6.16)$ & 0.832 \\
\hline $\mathrm{HbA} 1 \mathrm{c} \geq 6.5$ and no history of DM, IMV & & & $6.36(2.02-20.85)$ & 0.002 \\
\hline $\mathrm{HbA} 1 \mathrm{c} \geq 6.5$ and history of $\mathrm{DM}, \mathrm{IMV}$ & & & $3.66(1.38-10.17)$ & 0.011 \\
\hline
\end{tabular}

$\mathrm{HbA1c}$ and variables with a p value $<0.05$ in univariate analysis were included in multivariate analysis. Of 350 included patients, 44 have been previously reported [1] $B M I$ body mass index, SAPS simplified acute physiology score, IMV invasive mechanical ventilation, HbA1c glycated hemoglobin, DM diabetes mellitus, OR odds ratio, $\mathrm{Cl}$ confidence interval, Ref reference category

common than DM, chronic dysglycemia as defined by an HbA1c $\geq 5.7 \%$ was the predominant factor in our cohort of critically ill COVID-19 patients. We still did not find a strong association between elevated HbA1c and ICU/ hospital mortality, but in the subgroup of patients requiring mechanical ventilation, mortality was significantly increased in patients with $\mathrm{HbAlc} \geq 6.5 \%$. This is in line with other cohorts of COVID-19 patients, showing that admission blood glucose may be a more relevant predictor for mortality than HbA1c [3]. Admission blood glucose may be interpreted as a biomarker of systemic inflammation on admission, whereas HbA1c represents a marker of glucose control of the past three months [4, 5]. Unfortunately, blood glucose measurements during ICU admission were not recorded in our registry. Furthermore, follow-up of reported patients would be necessary to confirm diagnosis of previously unrecognized diabetes mellitus by admission HbA1c. We also have 
to acknowledge a potential selection bias for patients in whom HbA1c was measured. BMI has recently been reported as an important risk factor for severe COVID19 [3]. Though we could establish a weak correlation between BMI and HbA1c, we want to emphasize that prediabetes/and diabetes may exist independently and thus remain an independent risk factor for ICU admission in COVID-19. A possible explanation may be a pathological inflammatory response in diabetic patients [6]. When exposed to an additional inflammatory stimulus, such as mechanical ventilation on top of COVID-19, outcome may be impaired. This finding warrants further research in terms of risk stratification at ICU admission.

\begin{abstract}
Acknowledgements
Collaborators and their Institutions: Bellmann Romuald, Andreas Peer, Ditlbacher Adelheid, Haßlacher Julia, Lehner Georg F. and Perschinka Fabian: Division of Intensive Care and Emergency Medicine, Department of Internal Medicine, Medical University Innsbruck (Austria). Hasibeder Walter: Department of Anesthesiology and Critical Care Medicine, Hospital St. Vinzenz Zams (Austria). Krismer Christoph: Department of Internal Medicine, Hospital St. Vinzenz Zams (Austria). Pechlaner Agnes: Department of Internal Medicine, Hospital St. Vinzenz Zams (Austria). Eschertzhuber Stephan: Department of Anesthesia and Intensive Care Medicine, Hospital Hall (Austria). Zagitzer-Hofer Stefanie: Department of Anesthesia and Intensive Care Medicine, Hospital Hall (Austria). Foidl Eva: Department of Anesthesia and Intensive Care Medicine, Hospital Kufstein (Austria). Weilguni Isabella: Department of Anesthesia and Intensive Care Medicine, Hospital Kufstein (Austria). Haslauer-Mariacher Stefanie: Department of Anesthesia and Intensive Care Medicine, Hospital Kufstein (Austria). Kalenka Armin: Department of Anesthesia and Intensive Care Medicine, Hospital Kufstein (Austria). Ribitsch Alexandra: Department of Internal Medicine, Hospital Lienz (Austria). Mayr Andreas: Department of Anesthesia and Intensive Care Medicine, Hospital Lienz (Austria). Ladner Eugen: Department of Anesthesia and Intensive Care Medicine, Hospital Reutte (Austria), Heiner Tatjana: Department of Anesthesia and Intensive Care Medicine, Hospital Reutte (Austria). Mayr-Hueber Bernhard: Department of Anesthesia and Critical Care Medicine, Hospital Schwaz (Austria). Stögermüller Birgit: Department of Anesthesia and Critical Care Medicine, Hospital Schwaz (Austria). Kirchmair Lukas: Department of Anesthesia and Critical Care Medicine, Hospital Schwaz, (Austria). Reitter Bruno: Department of Anesthesia and Intensive Care Medicine, Hospital St. Johann in Tyrol (Austria). Potocnik Miriam Department of Anesthesia and Intensive Care Medicine, Hospital St. Johann in Tyrol (Austria). Mathis Simon: Department of Anesthesia and Critical Care Medicine, Medical University Innsbruck (Austria). Fiala Anna: Department of Anesthesia and Critical Care Medicine, Medical University Innsbruck (Austria). Brunner Jürgen: Department of Pediatrics, Medical University Innsbruck, (Austria). Claudius Thomé: Department of Neurosurgery, Medical University Innsbruck (Austria).
\end{abstract}

\section{Authors' contributions}

SJK, TM and MJ collected data and wrote the manuscript. DF, CPH, and the collaborators collected data for this study. All authors read and approved the final manuscript.

\section{Funding}

Funding for this study was provided by the Tyrolean Government.
Availability of data and material

No data are publicly available at this time.

\section{Declarations}

\section{Ethics approval and consent to participate}

This study was approved by the ethics committee of the Medical University Innsbruck (\# 1099/2020). Informed consent or post hoc informed consent was obtained.

\section{Consent for publication}

Not applicable-manuscript contains no individual patient data.

\section{Competing interests}

The authors declare that they have no competing interests.

\section{Author details}

${ }^{1}$ Division of Intensive Care and Emergency Medicine, Department of Internal Medicine, Medical University Innsbruck, Anichstrasse 35, 6020 Innsbruck, Austria. ${ }^{2}$ Department of Anesthesia and Critical Care Medicine, Medical University Innsbruck, Innsbruck, Austria. ${ }^{3}$ Department of Neurosurgery, Medical University Innsbruck, Innsbruck, Austria.

Received: 26 May 2021 Accepted: 12 August 2021

Published online: 19 August 2021

\section{References}

1. Klein SJ, Fries D, Kaser S, Mathis S, Thome C, Joannidis M. Unrecognized diabetes in critically ill COVID-19 patients. Crit Care. 2020;24(1):406.

2. Klein SJ, Bellmann R, Dejaco H, Eschertzhuber S, Fries D, Furtwangler W, Gasteiger L, Hasibeder W, Helbok R, Hochhold C, et al. Structured ICU resource management in a pandemic is associated with favorable outcome in critically ill COVID19 patients. Wien Klin Wochenschr. 2020;132(21-22):653-63.

3. Cariou B, Hadjadj S, Wargny M, Pichelin M, Al-Salameh A, Allix I, Amadou C, Arnault G, Baudoux F, Bauduceau B, et al. Phenotypic characteristics and prognosis of inpatients with COVID-19 and diabetes: the CORONADO study. Diabetologia. 2020;63(8):1500-15.

4. Schlesinger S, Neuenschwander M, Lang A, Pafili K, Kuss O, Herder C, Roden M. Risk phenotypes of diabetes and association with COVID-19 severity and death: a living systematic review and meta-analysis. Diabetologia. 2021;64(7):1480-91.

5. Umpierrez GE, Isaacs SD, Bazargan N, You X, Thaler LM, Kitabchi AE. Hyperglycemia: an independent marker of in-hospital mortality in patients with undiagnosed diabetes. J Clin Endocrinol Metab. 2002;87(3):978-82.

6. Apicella M, Campopiano MC, Mantuano M, Mazoni L, Coppelli A, Del Prato S. COVID-19 in people with diabetes: understanding the reasons for worse outcomes. Lancet Diabetes Endocrinol. 2020;8(9):782-92.

\section{Publisher's Note}

Springer Nature remains neutral with regard to jurisdictional claims in published maps and institutional affiliations. 